\title{
ON THE STAR-CONSTRUCTION FOR TOPOLOGICAL 4-MANIFOLDS
}

\author{
PETER TEICHNER
}

\begin{abstract}
We discuss existence and uniqueness of the *-construction which reverses the Kirby-Siebenmann invariant of a topological 4-manifold while fixing the homotopy type. In particular, we point out an error in Theorem 10.3 of [4] where a certain $*$-partner was mistakenly proven not to exist. The existence of this 4-manifold will follow from our Theorem 1 which proves that for fundamental group $\mathbb{Z} / 2$ all possible isometries of the equivariant intersection form are realized by homeomorphisms.
\end{abstract}

\section{INTRODUCTION}

One of the main surprises of Mike Freedman's classification of closed simply connected topological 4-manifolds in [3] was the existence of a manifold homotopy equivalent to $\mathbb{C P}^{2}$ but not homeomorphic to the same. In [4], Freedman and Quinn denote this manifold by $* \mathbb{C P}^{2}$ and use it to construct many more $*$-partners of 4-manifolds. More precisely, in [4, Thm.10.3] the authors prove that under the assumption that the universal covering of $\mathrm{M}$ is not spin (and the fundamental group is good) there exists a decomposition

$$
M \# * \mathbb{C P}^{2} \cong * M \# \mathbb{C P}^{2}
$$

which preserves the decomposition of $\pi_{2}$. By construction, $M$ and $* M$ are homotopy equivalent and have opposite Kirby-Siebenmann invariants.

Freedman and Quinn proceed to define $* M:=M$ if the universal covering of $M$ is spin because they claim that in this case a decomposition as above does not exist, see the last sentence in [4, Thm.10.3(1)]. However, we will show that this sentence cannot be correct by proving the following result. (It is understood that a decomposition (D) always preserves the decomposition of $\pi_{2}$.)

Proposition 1. Let $E$ be the quotient of $S^{2} \times S^{2}$ by the free involution sending $(x, y)$ to $(-x,-y)$. Then a decomposition $(D)$ as above exists for $M:=E$.

We would like to remark that in [12] Richard Stong found independently a mistake in the proof of [4, Thm.10.3] and gave a corrected statement which confirms our result on the existence of $* E$. Our methods differ considerably from those of Freedman, Quinn and Stong in that we make use of a modified surgery approach

1991 Mathematics Subject Classification. Primary: 57N13 Secondary: 55N22.

Supported by a Feodor Lynen Fellowship from the Humboldt foundation 
developed by Matthias Kreck in [11]. Proposition 1 will be implied by the following result which seems interesting for its own sake.

Theorem 1. Let $M_{1}$ and $M_{2}$ be closed oriented 4-manifolds with fundamental group $\mathbb{Z} / 2$ and with the same Kirby-Siebenmann invariants. Then any isometry $\pi_{2} M_{1} \rightarrow \pi_{2} M_{2}$ of intersection forms which preserves the $k$-invariant is induced by an orientation preserving homeomorphism.

Ian Hambleton pointed out to me that this result also follows from the classical surgery sequence, the homotopy theory developed in $[5]$ and the classification result in [6]. Since this path of proof is very long it seems interesting to present the completely different approach via Kreck's modified surgery technique.

Let me mention the following subtlety in the definition of $* M$ : In [7] we prove the existence of a manifold $R$ homotopy equivalent to $\mathbb{R P}^{4}$ but with nontrivial Kirby-Siebenmann invariant. We also show that $R \# \mathbb{C P}^{2}$ is not homeomorphic to $\mathbb{R} \mathbb{P}^{4} \# * \mathbb{C P}^{2}$ which means that Theorem 1 is not true in the nonoriented case and that $R$ is not a $*$-partner of $\mathbb{R P}^{4}$ in the sense of admitting a decomposition (D). However, in the classification of nonoriented 4-manifolds with fundamental group $\mathbb{Z} / 2$ (see [7]), $R$ plays a similar role as $* \mathbb{C P}^{2}$ does in the simply connected case. Similarly, in the known classification results for other fundamental groups (see [6] or [15]) it is not necessary to know that a decomposition (D) exists as long as one knows that one can reverse the Kirby-Siebenmann invariant while fixing the homotopy type of a given manifold. It seems safe to make the following

Definition. Set $* M:=M$ if there exists no 4-manifold in the homotopy type of $M$ which has the opposite Kirby-Siebenmann invariant.

It is wellknown that this definition applies if $M$ is a spin manifold because in this case the homotopy type of $M$ determines the Kirby-Siebenmann invariant by the formula (see [10])

$$
k s(M) \equiv \sigma(M) / 8 \quad \bmod 2
$$

where $\sigma(M)$ denotes the signature of the intersection form on $H_{2}(M ; \mathbb{Z})$.

It seemed not to be known whether our definition applies for any nonspin 4manifold. The following examples answer this question.

Proposition 2. If $M$ is either the nontrivial $S^{3}$-bundle over $S^{1}$ or the nontrivial orientable $S^{2}$-bundle over a surface of genus $>0$ then any manifold homotopy equivalent to $M$ has trivial Kirby-Siebenmann invariant. In fact, it is stably homeomorphic to $M$, i.e. homeomorphic up to connected sum with copies of $S^{2} \times S^{2}$.

Let me now turn to the uniqueness question of the $*$-construction which is certainly one of the most desirable properties. If the fundamental group is good and the universal covering of $M$ is not spin, Stong shows in [13] that the decomposition (D) determines $* M$ uniquely. (In the simply connected case this follows directly from Freedman's classification result [3].) Unfortunately, the following example shows that such a uniqueness statement cannot hold in general. First note that for $M:=E \# * E, E$ as in Proposition 1, the two manifolds $E \# E$ and $* E \# * E$ both satisfy a decomposition (D). Therefore, uniquess of the $*$-construction fails by the following result.

Proposition 3. The manifolds E\#E and $* E \# * E$ are not stably homeomorphic. 
I would like to thank my former advisor Matthias Kreck for helpful discussions concerning Theorem 1.

\section{Proofs of Propositions 2 and 3}

Propositions 2 and 3 are consequences of Kreck's stable classification theorem (see [11] or [15]). We will summarize the relevant definitions and results. Let $M$ be a manifold with fundamental group $\pi$ and universal covering spin. Choosing a 2-equivalence $u: M \rightarrow K(\pi, 1)$, the homotopy fibration $\widetilde{M} \stackrel{p}{\rightarrow} M \stackrel{u}{\rightarrow} K(\pi, 1)$ induces an exact sequence

$$
0 \rightarrow H^{2}(\pi ; \mathbb{Z} / 2) \stackrel{u^{*}}{\longrightarrow} H^{2}(M ; \mathbb{Z} / 2) \stackrel{p^{*}}{\longrightarrow} H^{2}(\widetilde{M} ; \mathbb{Z} / 2) .
$$

Since $0=w_{2} \widetilde{M}=p^{*}\left(w_{2} M\right)$, there exists a unique element $w_{2} \in H^{2}(\pi ; \mathbb{Z} / 2)$ with $w_{2} M=u^{*}\left(w_{2}\right)$. Clearly there also is a unique element $w_{1} \in H^{1}(\pi ; \mathbb{Z} / 2)$ with $w_{1} M=u^{*}\left(w_{1}\right)$. The triple $\left(\pi, w_{1}, w_{2}\right)$ is determined by $M$ up to automorphisms of $\pi$. We call the isomorphism class of this triple the $w$-type of $M$ and define the concept of (topological) $\left(\pi, w_{1}, w_{2}\right)$-bordism groups as follows. The classes $w_{1}, w_{2}$ give a fibration $\left(w_{1}, w_{2}\right): K(\pi, 1) \rightarrow K(\mathbb{Z} / 2,1) \times K(\mathbb{Z} / 2,2)$ and we can form the pullback

$$
\begin{array}{ccc}
B\left(\pi, w_{1}, w_{2}\right) & K(\pi, 1) \\
\xi\left(\pi, w_{1}, w_{2}\right) \downarrow & \downarrow\left(w_{1}, w_{2}\right) \\
B T O P & \stackrel{w_{1}(\gamma) \times w_{2}(\gamma)}{\longrightarrow} K(\mathbb{Z} / 2,1) \times K(\mathbb{Z} / 2,2)
\end{array}
$$

where $\gamma$ denotes the stable universal micro-bundle over $B T O P$, the classifying space of stable micro-bundles. Now the bordism groups

$$
\Omega_{n}\left(\pi, w_{1}, w_{2}\right):=\Omega_{n}\left(\xi\left(\pi, w_{1}, w_{2}\right)\right)
$$

can be defined as in [14]. They consist of bordism classes of triangles

$$
\begin{array}{cc}
\tilde{\nu} & B\left(\pi, w_{1}, w_{2}\right) \\
M & \downarrow \xi\left(\pi, w_{1}, w_{2}\right) \\
\underset{\nu}{\longrightarrow} & B T O P
\end{array}
$$

where $M$ is a closed manifold and $\nu$ is the stable normal Gauß map of $M$ given by some embedding into Euclidean space. The map $\tilde{\nu}$ is called a $\left(\pi, w_{1}, w_{2}\right)$-structure on $M$. If $\tilde{\nu}$ is a 2-equivalence, it is called a normal 1-smoothing. The following result is contained in [11].

Theorem. Two closed 4-manifolds with the same $w$-type $\left(\pi, w_{1}, w_{2}\right)$ are stably homeomorphic if and only if they admit $\left(\pi, w_{1}, w_{2}\right)$-bordant normal 1-smoothings. Moreover, any element in $\Omega_{n}\left(\pi, w_{1}, w_{2}\right)$ can be represented by a 4-manifold with w-type $\left(\pi, w_{1}, w_{2}\right)$ together with a normal 1-smoothing.

We remark that two such normal 1-smoothings always differ by a fibre homotopy equivalence of the fibration $\xi\left(\pi, w_{1}, w_{2}\right)$. Therefore, a 4-manifold with $w$-type $\left(\pi, w_{1}, w_{2}\right)$ which is $\left(\pi, w_{1}, w_{2}\right)$-zero-bordant can never be stably homeomorphic to a 4-manifold which does not bound in $\Omega_{4}\left(\pi, w_{1}, w_{2}\right)$.

This applies in particular to the manifolds $E \# E$ and $* E \# * E$ of Proposition 3 . Note 
that $E$ is an $S^{2}$-bundle over $\mathbb{R} \mathbb{P}^{2}$ and the corresponding $D^{3}$-bundle is a $\left(\mathbb{Z} / 2,0, x^{2}\right)$ bordism (here $x \in H^{1}(\mathbb{Z} / 2 ; \mathbb{Z} / 2)$ is a generator). The boundary connected sum of two copies of it provides a $\left(\mathbb{Z} / 2 * \mathbb{Z} / 2,0, x_{1}^{2}+x_{2}^{2}\right)$-bordism for $E \# E$. We will prove below that $* E \# * E$ is not zero in this bordism group which will finish the proof of Proposition 3.

But first we need to describe a method for computing the above bordism groups. The first step is to note that for any fibration $\xi: B \rightarrow B T O P$ the Pontrjagin-Thom construction gives an isomorphism

$$
\Omega_{n}(\xi) \cong \pi_{n}^{s t}(M \xi)
$$

where $M \xi$ is the Thom spectrum for $\xi$ with its bottom cell in dimension zero. In all the cases considered in this paper there exists a vector bundle $E$ over $K(\pi, 1)$ such that $w_{i}(E)=w_{i}$ for $i=1,2$. Then the fibration $\xi\left(\pi, w_{1}, w_{2}\right)$ is fibre homotopy equivalent $[15$, Ch.5] to

$$
\xi:=\gamma \oplus E: B:=B \operatorname{SpinTOP} \times K(\pi, 1) \longrightarrow B T O P
$$

where $\gamma$ is the universal spin micro-bundle over BSpinTOP. But this implies that we have a homotopy equivalence

$$
M \xi \simeq M \gamma \wedge M E
$$

and thus get an Atiyah-Hirzebruch spectral sequence (with respect to the homology theory associated to the spectrum $M \gamma$ )

$$
E_{p, q}^{2}=H_{p}\left(M E ; \Omega_{q}^{\text {SpinTOP }}\right) \Longrightarrow \Omega_{p+q}^{\text {SpinTOP }}(M E) \cong \Omega_{p+q}(\xi) .
$$

Finally, using the Thom isomorphism (for ordinary homology), the $E^{2}$-term becomes $H_{p}\left(\pi ; \Omega_{q}^{\text {SpinTOP }}\right)$ where the coefficients are twisted via $w_{1}$. This also implies (compare [16]) that the $d_{2}$-differentials for $q \leq 2$ are dual to the homomorphisms sending $x \in H^{p}(\pi ; \mathbb{Z} / 2)$ to $S q^{2}(x)+S q^{1}(x) \cdot w_{1}+x \cdot w_{2}$. This information implies the following

Lemma 2. The signature and Kirby-Siebenmann invariant induce an isomorphism

$$
\Omega_{4}\left(\mathbb{Z} / 2,0, x^{2}\right) \cong 8 \cdot \mathbb{Z} \times \mathbb{Z} / 2 .
$$

Moreover, together with an additional $\mathbb{Z} / 2$-valued invariant they induce an isomorphism

$$
\Omega_{4}\left(\mathbb{Z} / 2 * \mathbb{Z} / 2,0, x_{1}^{2}+x_{2}^{2}\right) \cong 8 \cdot \mathbb{Z} \times \mathbb{Z} / 2 \times \mathbb{Z} / 2 .
$$

Now fix $\left(\mathbb{Z} / 2,0, x^{2}\right)$-structures on $E$ and $* E$ which are normal 1-smoothings and always use their connected sum as $\left(\mathbb{Z} / 2 * \mathbb{Z} / 2,0, x_{1}^{2}+x_{2}^{2}\right)$-structures on the connected sum manifolds. Consider the two inclusions $f_{i}: \mathbb{Z} / 2 \rightarrow \mathbb{Z} / 2 * \mathbb{Z} / 2$. There are induced homomorphisms

$$
f_{i *}: \Omega_{4}\left(\mathbb{Z} / 2,0, x^{2}\right) \longrightarrow \Omega_{4}\left(\mathbb{Z} / 2 * \mathbb{Z} / 2,0, x_{1}^{2}+x_{2}^{2}\right)
$$

which send $* E$ to $E \# * E$ respectively $* E \# E$. A comparison of the corresponding spectral sequences implies that these two elements are generators of the two $\mathbb{Z} / 2$ summands in this bordism group. Therefore, the sum of these two elements, which is bordant to $* E \# * E$, is nontrivial.

Now let $\pi(g)$ be the fundamental group of a surface of genus $g \geq 0$. Then 
$H^{2}(\pi(g) ; \mathbb{Z} / 2)=\mathbb{Z} / 2$ so there is a unique orientable but nonspin $w$-type for $\pi(g)$. Similarly, $H^{1}(\mathbb{Z} ; \mathbb{Z} / 2)=\mathbb{Z} / 2, H^{2}(\mathbb{Z} ; \mathbb{Z} / 2)=0$ implies that there is a unique nonspin $w$-type for fundamental group $\mathbb{Z}$. Proposition 2 immediately follows from the following

Theorem 3. If $M$ is one of the manifolds in Proposition 2, the bordism groups of its w-type are

$$
\Omega_{4}(\mathbb{Z}, \neq 0,0) \cong \mathbb{Z} / 2 \text { and } \Omega_{4}(\pi(g), 0, \neq 0) \cong 8 \cdot \mathbb{Z} \times \mathbb{Z} / 2
$$

where the isomorphisms are given by the Kirby-Siebenmann invariant respectively the signature and the Kirby-Siebenmann invariant. Moreover, the intersection form on $\pi_{2}$ induces (additive) isomorphisms with Wall's L-groups

$$
\Omega_{4}\left(\pi, w_{1}, w_{2}\right) \cong L_{4}\left(\pi, w_{1}\right) .
$$

In particular, the stable intersection form on $\pi_{2}$ determines the stable homeomorphism type of 4-manifolds in the w-type of $M$.

Proof. The computation of the bordism groups is a straightforward application of the methods described above. The second part splits naturally into two cases.

Case 1: $M$ is the nontrivial $S^{3}$-bundle over $S^{1}$.

Then $M$ and $M \# E_{8}$ constitute all stable homeomorphism classes in the $w$-type $(\mathbb{Z}, \neq 0,0)$. Therefore, $\pi_{2}$ is always a stably free $\mathbb{Z}[\mathbb{Z}]$-module and the intersection form is nondegenerate. Since it uniquely determines a quadratic refinement, the class in $L_{4}(\mathbb{Z}, \neq 0)$ is welldefined. But one knows from [17] that this $L$-group is isomorphic to $\mathbb{Z} / 2$, generated by $E_{8} \otimes \mathbb{Z}[\mathbb{Z}]=\pi_{2}\left(M \# E_{8}\right)$.

Case 2: $M$ is the nontrivial orientable $S^{2}$-bundle over a surface of genus $g$.

Then $\pi_{2}(M)=\mathbb{Z}$ is not a free $\pi(g)$-module but a trivial module. Also the intersection form vanishes for $M$. We claim that any 4-manifold with the given $w$-type has the trivial module $\mathbb{Z}$ as the radical of its intersection form on $\pi_{2}$ and the form on the quotient is nondegenerate on a stably free $\pi(g)$-module with a unique quadratic refinement. This defines our map to $L_{4}(\pi(g), 0)$. To prove our claim, let $X \rightarrow M$ be a degree 1 normal map with signature 0 and $k s(X) \neq 0$ (which always exists by $[10$, p.329] if $M$ is orientable but nonspin). Then $M \# W$ and $X \# W, W$ a simply connected closed spin 4-manifold, constitute all stable homeomorphism classes in the $w$-type $(\pi(g), 0, \neq 0)$. But the kernel on $\pi_{2}$ of a degree 1 normal map is always a nondegenerate form on a stably free module which proves our claim for $X$ and thus for all manifolds in our $w$-type. Using the fact that the addition in the bordism group is realized by connected sum along the 1-skeleton [11] of manifolds with the correct $w$-type, it is not hard to check that our map to $L_{4}$ is a homomorphism. It is well known [1] that for surface groups the assembly map gives an isomorphism $\left(w_{1}=0\right.$ in all cases and $g>0$ as before)

$$
L_{4}(\pi(g)) \cong L_{4}(1) \times L_{2}(1) \cong 8 \cdot \mathbb{Z} \times \mathbb{Z} / 2
$$

induced by the signature and the codimension 2 Arf invariant. But this Arf invariant equals $\sigma(N) / 8+k s(N)$ if $N$ has the correct $w$-type. This finishes our proof. 


\section{Proof of Theorem 1}

Our proof will not use the more difficult classification of oriented 4-manifolds with fundamental group $\mathbb{Z} / 2$ in $[6]$ in terms of the intersection form on $H_{2}(. ; \mathbb{Z} / 2)$. Instead, we will work along the lines of the method of Kreck in [11, Ch.13] who proved the analogue of Theorem 1 for certain odd order fundamental groups, e.g. odd order groups in which every Sylow subgroup has an abelian normal subgroup with cyclic quotient. However, our bordism calculations are much more involved in that we have to prove the existence of a nontrivial $d_{3}$-differential in a certain Atiyah-Hirzebruch spectral sequence.

Let me now show how Theorem 1 implies Proposition 1: Let $M$ be a closed nonspin 4-manifold such that $\pi_{1}(M)$ is finite with a 2-Sylow subgroup of periodic cohomology. Then a result of [8] on the Wall obstruction for closed manifold surgery problems implies the existence of a manifold $M^{\prime}$ homotopy equivalent to $M$ but with opposite Kirby-Siebenmann invariant. For details of this argument see [15, Ch.8]. In particular, we get a 4 -manifold $E^{\prime} \simeq E$ with $k s\left(E^{\prime}\right) \neq 0$. This gives a homotopy equivalence

$$
E \# * \mathbb{C P}^{2} \longrightarrow E^{\prime} \# \mathbb{C P}^{2}
$$

which preserves the decomposition on $\pi_{2}$. Since any homotopy equivalence preserves the $k$-invariant, Theorem 1 implies that the same decomposition of $\pi_{2}$ is induced by a homeomorphism. This proves Proposition 1 and shows the existence of $* E$. Note that by construction $E^{\prime}$ and $* E$ are homotopy equivalent and a second application of Theorem 1 proves that they are homeomorphic.

Proof of Theorem 1. In order to control $\pi_{2}$ we have to climb up one more step in the Postnikov tower of the stable normal Gauß map of an oriented 4-manifold $M$. To do so, let $X$ denote a $\mathrm{CW}$-complex with trivial homotopy groups in dimensions 3 and higher and admitting a 3-equivalence $u: M \rightarrow X$. Then $X$ is determined up to homotopy by $M$, in fact by the triple $\left(\pi_{1} M, \pi_{2} M, k(M)\right)$ where $\pi_{2} M$ is considered as a $\pi_{1} M$-module and $k(M) \in H^{3}\left(\pi_{1} M ; \pi_{2} M\right)$ is the first k-invariant of $M$ classifying the fibration

$$
K\left(\pi_{2} M, 2\right) \rightarrow X \rightarrow K\left(\pi_{1} M, 1\right) .
$$

Using obstruction theory, it is easy to see that any automorphism of the above triple is induced by a self-homotopy equivalence of $X$. In particular, given an isomorphism of triples

$$
\left(\pi_{1} M_{1}, \pi_{2} M_{1}, k\left(M_{1}\right)\right) \cong\left(\pi_{1} M_{2}, \pi_{2} M_{2}, k\left(M_{2}\right)\right)
$$

we may choose 3 -equivalences $u_{i}: M_{i} \rightarrow X$ such that $u_{1 *} \circ u_{2 *}^{-1}$ induces the isomorphism. If we assume further that this isomorphism is an isometry of intersection forms on $\pi_{2}$ and that $\pi_{1}$ is cyclic then by [5] we also have

$$
u_{1 *}\left[M_{1}\right]=u_{2 *}\left[M_{2}\right] \in H_{4}(X ; \mathbb{Z}) .
$$

Let $\xi: B S p i n T O P \times X \stackrel{\gamma \oplus L}{\longrightarrow} B S T O P$ be a fibration coming from the classifying map of the Whitney sum of the universal spin micro-bundle $\gamma$ and a complex line bundle $L$ over $X$ with $w_{2}(L)=u^{*}\left(w_{2} M\right)$. By construction, the stable normal Gauß map $\nu: M \rightarrow B S T O P$ lifts over $\xi$ and any such lift $\tilde{\nu}$ is called a normal2-structure of $M$. If $\tilde{\nu}$ is a 3 -equivalence, it is called a normal 2-smoothing. Note that the 
difference bundle $\nu(M)-u^{*}(L)$ is spin and thus we obtain a normal 2-smoothing of the form $\tilde{\nu}=(s, u)$ with $s$ some spin structure on this difference. We will use the following theorem from [11].

Theorem. Let $\pi_{1}$ be a good group with $L_{5}^{s}\left(\pi_{1}\right)=0$. If there exist $\xi$-bordant normal 2 -smoothings $\left(M_{i}, \tilde{\nu}_{i}\right)$ then there exists an orientation preserving homeomorphism $h: M_{1} \rightarrow M_{2}$ over $X$. In particular, $h$ induces the given isometry on $\pi_{2}$.

Noting that by $[17] L_{5}^{s}(\mathbb{Z} / 2)=0$, our Theorem 1 follows from the following

Proposition 4. Let $\pi_{1}=\mathbb{Z} / 2$. Then signature, Kirby-Siebenmann invariant and image of the fundamental class induce a monomorphism

$$
\left(\sigma, k s, u_{*}\right): \Omega_{4}(\xi) \longmapsto \mathbb{Z} \times \mathbb{Z} / 2 \times H_{4}(X ; \mathbb{Z}) .
$$

Proof. As in the previous section we make use of the Atiyah-Hirzebruch spectral sequence

$$
E_{p, q}^{2}=H_{p}\left(M L ; \Omega_{q}^{\text {SpinTOP }}\right) \Longrightarrow \Omega_{p+q}^{\text {SpinTOP }}(M L) \cong \Omega_{p+q}(\xi) .
$$

Since we are in the oriented case, we may use the Thom isomorphism to identify $H_{p}\left(M L ; \Omega_{q}^{S p i n T O P}\right)$ with $H_{p}\left(X ; \Omega_{q}^{\text {SpinTOP }}\right)$ (ordinary homology, no twisting). Then the $d_{2}$-differentials for $q \leq 2$ are dual to the homomorphisms sending $x \in$ $H^{p}(X ; \mathbb{Z} / 2)$ to $S q^{2}(x)+x \cdot w_{2}(L)$. Moreover, the boundary homomorphism $\Omega_{4}(\xi) \rightarrow$ $H_{4}(X ; \mathbb{Z})$ is just the image of the fundamental class under $u_{*}$ (see [16]) and controlling the signature means that we can work with the reduced theory $\widetilde{\Omega}_{4}(\xi)$. Therefore, our aim is to show that we have

$$
E_{3,1}^{\infty}=0 \text { and } E_{2,2}^{\infty}=\mathbb{Z} / 2 .
$$

This last $\mathbb{Z} / 2$ will then necessarily have to be detected by the Kirby-Siebenmann invariant. The homology of $X$ may be calculated using the Leray-Serre spectral sequence for the fibration $(*)$ once one knows the structure of $\pi_{2}:=\pi_{2} X \cong \pi_{2} M$ as a $\pi_{1}$-module and the $k$-invariant. But for fundamental group $\mathbb{Z} / 2$ this is easy since by [2] any $\mathbb{Z}$-torsionfree $\mathbb{Z} / 2$-module has a unique decomposition as a direct sum of only three possible building blocks, namely $\mathbb{Z}^{+}, \mathbb{Z}^{-}$and $A$. Here $A:=\mathbb{Z}[\mathbb{Z} / 2]$ is the free module of rank 1 and $\mathbb{Z} / 2$ acts trivially on $\mathbb{Z}^{+}$and as -1 on $\mathbb{Z}^{-}$. Therefore, the stable information on $\pi_{2}$ which one gets by the methods of the previous section imply the unstable isomorphism (see also [5])

$$
\pi_{2} \cong \mathbb{Z}^{-} \oplus \mathbb{Z}^{-} \oplus A^{n} \text { and } 0 \neq k \in H^{3}\left(\mathbb{Z} / 2 ; \pi_{2}\right)=\mathbb{Z} / 2 \times \mathbb{Z} / 2 .
$$

Since the nontrivial $k$-invariant implies a nontrivial differential $d_{3}$ in the Leray-Serre spectral sequence, it follows that for the generator $x \in H^{1}(X ; \mathbb{Z} / 2)=\mathbb{Z} / 2$ we have the relation $x^{3}=0$. Moreover, a basis for $H^{2}(X ; \mathbb{Z} / 2)$ is given by

$$
\left\{x^{2}, a, z_{1}, \ldots, z_{n}\right\}
$$

with $a$ restricting to the $\mathbb{Z}^{-} \oplus \mathbb{Z}^{-}$part of $\pi_{2}^{*}$ in the kernel of $d_{3}$ and $z_{i}$ corresponding to the augmentation homomorphism of the $i$-th $A$-factor. We note also the relations $x^{i} \cdot z_{j}=0 \quad \forall i>0$ and the linear independence of all $z_{i}^{k} \cdot z_{j}^{l}, a^{k} \cdot z_{j}^{l}$ and $a^{k} \cdot x^{m} \quad \forall k, l \geq$ $0, m<3$. 
Corresponding to the three possible $w_{2}$-types of $M$, i.e. $\widetilde{M}$ nonspin, $M$ spin and $\widetilde{M}$ spin but $M$ nonspin, there are three possibilities for $w_{2}(L)$ (after a change of basis of $A^{n}$ ):

(I) $w_{2}(L)=x^{2}+z_{1}$.

(II) $w_{2}(L)=0$.

(III) $w_{2}(L)=x^{2}$.

In all these cases we have $E_{3,1}^{3}=0$ because the differential

$$
d_{2}: H_{5}(X ; \mathbb{Z}) \rightarrow H_{3}(X ; \mathbb{Z} / 2)
$$

is onto: Since $H_{5}(X ; \mathbb{Z})$ consists only of 2 -torsion, it is enough to show that the composition

$$
S q^{1} \circ\left(S q^{2}+w_{2}(L)\right): H^{3}(X ; \mathbb{Z} / 2) \rightarrow H^{6}(X ; \mathbb{Z} / 2)
$$

is injective. But $H^{3}(X ; \mathbb{Z} / 2)$ is generated by $a \cdot x$ which is mapped to $a^{2} \cdot x^{2} \neq 0$ under the above map. For $E_{2,2}^{3}$ we have to study the kernel of the map

$$
S q^{2}+w_{2}(L): H^{2}(X ; \mathbb{Z} / 2) \rightarrow H^{4}(X ; \mathbb{Z} / 2) .
$$

From the above information on $H^{*}(X ; \mathbb{Z} / 2)$ we can easily deduce that for $w_{2}$-type I this kernel is generated by $x^{2}$ and $z_{1}$ whereas for $w_{2}$-types II and III it is generated solely by $x^{2}$. In these last cases we are done since a single element must survive to $E_{2,2}^{\infty}$ because of the Kirby-Siebenmann invariant. For $w_{2}$-type I we have to show that the differential $d_{3}: E_{5,0}^{3} \rightarrow E_{2,2}^{3}$ is nontrivial which will finish our proof. We concentrate on the important information by considering the commutative diagram of fibrations

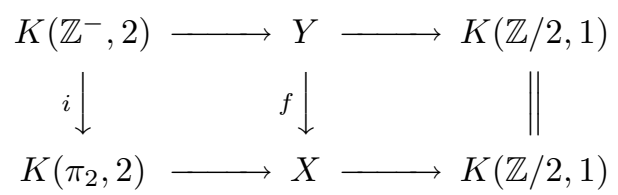

where $Y$ has nontrivial k-invariant and the map $i$ on the fibres is induced by the $\mathbb{Z} / 2$-homomorphism sending the generator of $\mathbb{Z}^{-}$to the sum $\kappa+(1-t) \cdot a_{1}$. Here $\kappa \in \mathbb{Z}^{-} \oplus \mathbb{Z}^{-}$reduces to the $k$-invariant and $a_{1}$ is the free generator of the first component in $A^{n}$ (which we decided to carry one part of $w_{2}(L)$ ). Also $t$ denotes the generator of $\mathbb{Z} / 2$. Since the class $x$ is the pullback of the generator in $H^{1}(\mathbb{Z} / 2 ; \mathbb{Z} / 2)$ we can denote $f^{*}(x)$ again by $x$ and it will generate $H^{1}(Y ; \mathbb{Z} / 2)$. We will now show the crucial property $f^{*}\left(z_{1}\right)=x^{2}$ : This follows from the commutative diagram of Gysin-sequences where we consider the universal coverings ( $\simeq$ fibres) of $X$ and $Y$ as $S^{0}$-bundles over $X$ respectively $Y$ (both having first Stiefel-Whitney class equal to $x$ ):

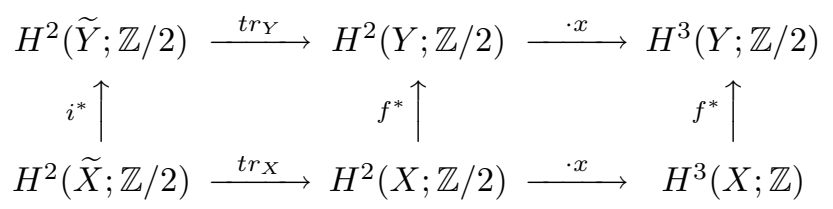

Since both $k$-invariants are nontrivial, the above multiplication with $x$ is identically zero. In $H^{2}(\widetilde{X} ; \mathbb{Z} / 2)$ we know that there exists a class $z$ corresponding to the nonequivariant homomorphism $A^{n} \rightarrow \mathbb{Z}$ which sends $a_{1}$ to $1, t \cdot a_{1}$ to 0 and also all the other free generators and their $t$-translates to 0 . Then $\operatorname{tr}_{X}(z)=z_{1}$ and $i^{*}(z)$ 
generates $H^{2}(\tilde{Y} ; \mathbb{Z} / 2)=\mathbb{Z} / 2$. Using the fact that $\operatorname{tr}_{Y}$ is an isomorphism and that $H^{2}(Y ; \mathbb{Z} / 2)$ is generated by $x^{2}$ we get

$$
f^{*}\left(z_{1}\right)=f^{*}\left(\operatorname{tr}_{X}(z)\right)=\operatorname{tr}_{Y}\left(i^{*}(z)\right)=x^{2} .
$$

This implies that the pullback under $f$ of the line bundle $L$ over $X$ gives the trivial bundle and thus we get an induced map

$$
\Omega_{*}^{\text {SpinTOP }}(Y) \cong \Omega_{*}^{\text {SpinTOP }}\left(M f^{*}(L)\right) \longrightarrow \Omega_{*}^{\text {SpinTOP }}(M L)=\Omega_{*}(\xi) .
$$

Because the map $f_{*}: E_{2,2}^{3}\left(M f^{*}(L)\right) \rightarrow E_{2,2}^{3}(M L)$ is nontrivial it suffices to show that the $d_{3}$-differential in question is nontrivial in the Atiyah-Hirzebruch spectral sequence for $\Omega_{*}^{\operatorname{SpinTOP}}(Y)$. To do so, we have to ask the reader for some more patience because we have to make two more comparisons to reduce the problem to a $d_{2}$-differential where it finally will be solved. For the first comparison, let $\eta$ be the real line bundle over $Y$ with $w_{1}(\eta)=x$ and thus $S(\eta)=\widetilde{Y}$. Writing $T(\eta)$ for its Thom space we get a cofibration

$$
\tilde{Y} \stackrel{p}{\rightarrow} Y \stackrel{q}{\rightarrow} T(\eta) .
$$

This cofibration induces long exact sequences in any (generalized) homology theory. We conclude that $q_{*}$ is an isomorphism in $H_{2}(; \mathbb{Z} / 2)$ and $H_{5}(; g z)$ and thus it suffices to show the nontriviality of our $d_{3}$-differential in the Atiyah-Hirzebruch spectral sequence for $\Omega_{*}^{\operatorname{SpinTOP}}(T(\eta))$. For the second comparison, we use the cofibration (compare [9])

$$
T\left(p^{*}(\eta)\right) \rightarrow T(\eta) \rightarrow T(\eta \oplus \eta) .
$$

Since $p^{*}(\eta)$ is the trivial line bundle over $\widetilde{Y}$ we get the suspension isomorphism

$$
\Omega_{4}\left(T\left(p^{*}(\eta)\right)\right) \cong \Omega_{3}(\tilde{Y}) .
$$

This group is easily checked to be trivial by the usual spectral sequence (only $d_{2}$-differentials are needed here!) We may conclude that $\Omega_{4}^{\text {SpinTOP }}(T(\eta))$ maps injectively into $\Omega_{4}^{\operatorname{SpinTOP}}(T(\eta \oplus \eta))$. Since the filtration degree of an element in a spectral sequence can only go up, we are certainly done if $E_{2,2}^{3}(T(\eta \oplus \eta))=0$. But this follows from the injectivity of the map

$$
S q^{2}: H^{2}(T(\eta \oplus \eta) ; \mathbb{Z} / 2) \rightarrow H^{4}(T(\eta \oplus \eta) ; \mathbb{Z} / 2)
$$

which sends the Thom class $u$ to $S q^{2}(u)=w_{2}(\eta \oplus \eta) \cdot u=x^{2} \cdot u \neq 0$.

\section{REFERENCES}

1. S. E. Cappell. Unitary nilpotent groups and hermitian K-theory. Bulletin of the AMS 80, (1974) 1117-1122

2. C. W. Curtis, I. Reiner. Representation Theory of Finite Groups and Associative Algebras. Series of Texts and Monographs XI, Wiley and sons 1962.

3. M. Freedman. The Topology of 4-dimensional Manifolds. J. of Diff. Geom. 17 (1982) 357-453.

4. M. Freedman, F. Quinn. Topology of 4-Manifolds. Princeton Mathematical Series 39, Princeton University Press 1990.

5. I. Hambleton and M. Kreck. On the Classification of Topological Four-Manifolds with Finite Fundamental Group. Math. Annalen 280 (1988) 85-104.

6. I. Hambleton and M. Kreck. Cancellation, Elliptic Surfaces and the Topology of certain Four-Manifolds. Preprint of the Max-Planck Institut für Mathematik Bonn 1991, to appear in the Journal für reine und angewandte Mathematik. 
7. I. Hambleton, M. Kreck, P. Teichner. Nonorientable 4-Manifolds with Fundamental Group of Order 2. Preprint 1992, to appear in the Transactions of the AMS.

8. I. Hambleton, R. J. Milgram, L. Taylor, B. Williams. Surgery with Finite Fundamental Group. Proc. London Math. Soc. (3)56 (1988) 349-379.

9. I. M. James. The Topology of Stiefel-Manifolds. London. Math. Soc. Lecture Note Series 24, Cambridge University Press 1976.

10. R. C. Kirby, L. C. Siebenmann. Foundational Essays on Topological Manifolds, Smoothings and Triangulations. Ann. of Math. Studies 88, Princeton University Press 1977.

11. M. Kreck. Surgery and Duality. To appear as a book in the Vieweg-Verlag, Wiesbaden. As a preprint of the Johannes-Gutenberg-Universität Mainz 1985 available under the title: $A n$ Extension of Results of Browder, Novikov and Wall about Surgery on Compact Manifolds.

12. R. Stong. Existence of $\pi_{1}$-Negligible Embeddings in 4-Manifolds. A Correction to Theorem 10. 5 of Freedman and Quinn. UCLA Preprint 1992.

13. R. Stong. Uniqueness of connected sum decompositions in dimension 4. UCLA Preprint 1992.

14. R. M. Switzer. Algebraic Topology-Homotopy and Homology. Die Grundlehren der math. Wissenschaften 212, Springer-Verlag 1970.

15. P. Teichner. Topological Four-Manifolds with Finite Fundamental Group. $\mathrm{PhD}$ Thesis at the University of Mainz, Germany. Verlag Shaker 1992.

16. P. Teichner. On the signature of four-manifolds with universal covering spin. Math. Annalen 295 (1993) 745-759.

17. C. T. C. Wall. Surgery on Compact Manifolds. London Math. Soc. Monographs 1, Academic Press 1970.

University of Mainz, SaARstr., 6500 Mainz,Germany

Current address: University of California, San Diego, 9500 Gilman Dr, La Jolla, CA, 92093

E-mail address: teichner@euclid.ucsd.edu 